\title{
The relationship between education and food consumption in the 1995 Australian National Nutrition Survey
}

\author{
Anthony Worsley*, Roswitha Blasche, Kylie Ball and David Crawford \\ School of Exercise and Nutrition Sciences, Deakin University, 221 Burwood Highway, Burwood, Victoria 3125 , \\ Australia
}

Submitted 16 June 2003: Accepted 25 November 2003

\begin{abstract}
Objective: To assess the relationship between education and the intake of a variety of individual foods, as well as groups of foods, for Australian men and women in different age groups.

Design: Cross-sectional national survey of free-living men and women.

Subjects: A sample of 2501 men and 2739 women aged 18 years and over who completed the National Nutrition Survey (NNS) 1995.

Methods: Information about the frequency of consumption of 88 food items was obtained using a food-frequency questionnaire in a nation-wide nutrition survey. Irregular and regular consumers of foods were identified according to whether they consumed individual foods less than or more than once per month. The relationship between single foods and an index of education (no post-school qualifications, vocational, university) was analysed via contingency table chi-square statistics for men and women. Food group variety scores were derived by assigning individual foods to conventional food group taxonomies, and then summing the dichotomised intake scores for individual foods within each food group. Two-way analyses of variance (education by age groups) were performed on food variety scores for men and women, separately.

Results: While university-educated men and women consumed many individual foods more regularly than less-educated people, they were less likely to be regular consumers of several meat products. The relationship between education and food consumption was less apparent when individual food scores were aggregated into food group scores. University-educated men and women exhibited higher scores on total food group variety than the other educational groups.

Conclusions: Higher education is associated with the regular consumption of a wider variety of foods. Aggregation of individual food consumption indices into food variety scores may mask the apparent effects of educational background on food consumption.
\end{abstract}

A large body of studies have demonstrated relationships between sociodemographic factors and food intake ${ }^{1-13}$. Among these, education is one factor that appears to have a fundamental influence on population food choice ${ }^{14,15}$. Studies of education and food choice are important since education, unlike gender, age or other demographic factors, is an achieved characteristic, and hence may be amenable to policy interventions. There are a number of reasons why education might influence food choice. First, education may open the way to differential access to food and health information. Second, social diffusion theory suggests that highly educated people generally take up innovations sooner than less-educated people ${ }^{16}$. For example, in the UK, foods and diets low in saturated fat were adopted by the tertiary-educated before others ${ }^{13}$. Third, social epidemiologists suggest that education enables people to rise up the social class hierarchy, allowing them greater power over outcomes in their lives, for example through higher incomes ${ }^{17}$. Davies ${ }^{18}$ and Ippolito $^{19}$ in particular have shown that tertiary education indices represent future-oriented belief systems that value self-control and prevention and downplay external fatalistic explanations of personal events.

Recent evidence shows that members of tertiaryeducated groups tend to know more about food and nutrition ${ }^{2,13,20}$. They also appear to have healthier dietary habits ${ }^{21-24}$. In contrast, lower education has been found to be associated with various indices of poor diet, including diets higher in fat density $9,25,26$ and the purchase of a restricted variety of fruit and vegetables ${ }^{27}$. However, the details of these relationships are unclear and tend to vary from study to study, perhaps because of differing study 
designs, different measurement levels (e.g. consumption of food groups vs. individual foods) and differing national contexts.

Other demographic factors are also associated with food consumption patterns ${ }^{1-13}$. Gender appears to have a pervasive influence. Women tend to take more responsibility for food selection and preparation than men ${ }^{3}$. They are thus likely to be more knowledgeable about nutrition $^{4,5}$ and more likely to acquire health-related knowledge than men ${ }^{6-8}$. It is, however, equally possible that the reason for women's heightened interest in and knowledge of nutrition information may be found in weight concerns. The finding that women are more likely to select low-fat foods than men ${ }^{8,9}$ may be reflective of concerns about weight control.

Age has also been shown to be associated with food and nutrient intake patterns ${ }^{10,11}$. As people age, they tend to consume more familiar foods ${ }^{11}$, even though these foods may be inadequate in addressing the physiological needs of the ageing body. Malnutrition in the older person may be caused by poor dietary intake. However, age effects on food intake are often confounded with those of education since many older people have had no extensive education $^{10}$.

Many dietary guidelines recommend the inclusion of a variety of foods in the diet, because nutrients that are important for health are not obtained from one single food or food group ${ }^{28}$. The Food Guide Pyramid is an example of a graphical depiction of food variety where individual foods are assigned to major food groups ${ }^{29}$. Grouping of individual foods in this way, however, masks the fact that some foods within one food group are less nutritious than others $^{30}$. Wholemeal bread would thus be grouped in the same category as white bread (cereal), and bacon, which is high in saturated fat, is included in the category 'meat' as are the lower-fat lean meats and liver. If being female, young and highly educated is indeed associated with the consumption of healthy foods and the grouping of foods has the effect of masking differences between foods that differ in nutritional value, then one would expect the association between demographic factors and food groups to be less clearly visible than the association between demographic factors and individual foods.

The current study distinguishes itself from past research by comparing associations of education with both individual foods and food groups. It is important in potentially helping to clarify some of the inconsistencies in the relationships between education and dietary habits referred to previously.

The main aim of this study was to examine the association of education with food intake. A subsidiary aim was to examine the effect of different ways of aggregating food intake (consumption of food groups and individual foods) on the association between education and food consumption. It was hypothesised that men and women with higher education would be more likely to report regular consumption of individual foods considered nutritious, but that relationships between education and consumption of food groups would be less consistent. Since gender and age are associated with food patterns, all analyses were conducted separately for these groups, and the interaction of age with education was examined for men and women.

\section{Method}

\section{Procedure}

Data from 5240 participants in the 1995 Australian National Health Survey (NHS) and the Australian National Nutrition Survey (NNS) 1995 were analysed ${ }^{31,32}$. Recruitment procedures for the 1995 NHS and NNS surveys are described in detail elsewhere ${ }^{31,33}$. Briefly, for the NHS, a stratified, multistage area sampling technique was used by the Australian Bureau of Statistics (ABS) to obtain a random, nation-wide sample of approximately 23800 households. Of households selected to participate, $91.5 \%$ responded, with a total of 57633 persons interviewed. Of those, 22562 were selected to participate in the NNS. The sample for the NNS was systematically selected from the NHS private dwelling sample covering urban and rural areas across all States and Territories of Australia. The NNS sub-sample was designed to provide desirable estimates of nutrient intake differences across groups (for example, national-level, state-level and regional estimates by age group and sex). A total of 13858 persons (61\% of those selected) participated in the NNS. Those on high incomes, unemployed people and people who were older than 59 years were more likely to decline participation than others. The present study uses data provided by those 5240 people aged 18 years and over (2501 men, 2739 women) who gave information about their post-school qualification and completed a food-frequency questionnaire (FFQ).

\section{Measures}

\section{Food intake}

The FFQ used in the NNS is a retrospective food-frequency questionnaire that provides semi-quantitative information on the longer-term consumption of 107 foods and beverages $^{33}$. For the purpose of this analysis, beverage items were excluded, leaving a total of 88 food items. The ABS had classified a person's record as unusable if more than 20 out of the 107 food lines were completed incorrectly. Almost 22\% of the sample did not return an FFQ; response increased with age for those aged over 20 years, and was lower for unmarried people than for married people. Only $2 \%$ or 237 of the FFQs that had been returned were deemed to be unusable by the ABS.

Respondents indicated average frequency of consumption of the 88 food items over the past 12 months on a 9-point scale: (1) never, or less than once a month, (2) 1-3 
times per month, (3) once per week, (4) 2-4 times per week, (5) 5-6 times per week, (6) once per day, (7) 2-3 times per day, (8) 4-5 times per day and (9) $6+$ times per day. Frequency of consumption was transformed into a dichotomous variable by defining a food as being 'regularly consumed' if it was consumed 1-3 times per month or more frequently, and as 'irregularly consumed' if it was consumed never or less than once per month. This definition has been used in a previous study by the authors $^{12}$.

There is no consensus as to how to group foods ${ }^{28}$. For the purpose of this study, food groups were created by assigning individual foods to conventional food group taxonomies. The following food groups were thus derived: fruit (apple or pear; orange, mandarin or grapefruit; banana; peach, nectarine, plum or apricot; mango or paw-paw; pineapple; grapes or berries; melon), vegetables (potato; pumpkin; sweet potato; peas; green beans; silverbeet, spinach; broccoli; cauliflower; Brussels sprouts, cabbage or coleslaw; carrots; zucchini; capsicum; sweet corn or corn on the cob; mushrooms; tomatoes; lettuce; celery, cucumber; onion or leeks; soybeans, tofu; baked beans; other beans, lentils; vegetable casserole; green/mixed salad in sandwich; side salad with meal; stir-fried or mixed vegetables), meat (mince dishes; mixed dishes with beef or veal; beef, veal - roast, chop or steak; mixed dishes with lamb; lamb - roast, chop or steak; mixed dishes with pork; pork - roast, chop or steak; sausage, frankfurter; bacon; ham; luncheon meats, salami; liver; other offal; mixed dishes with chicken, turkey or duck, etc.; chicken, turkey or duck - roast, steamed or barbecued), dairy (flavoured milk drink; milk as drink; milk on breakfast cereals; milk in hot beverages; cream or sour cream; ice cream; yoghurt, plain or flavoured (including fromage frais); cottage or ricotta cheese; cheddar and other cheeses; egg), cereals (white bread, toast or rolls; wholemeal/mixed-grain bread, toast or rolls; muffin, bagel or crumpet; dry or savoury biscuits; muesli; cooked porridge; breakfast cereal; rice; pasta), fish (canned tuna, salmon, sardines; fish, baked or grilled; fish fried; other seafood), spreads (jam, marmalade, syrup or honey; peanut butter or other nut spreads; vegemite, marmite or promite; mayonnaise dressing; oil and vinegar dressing), snacks (meat pie, sausage roll or other savoury pastry; pizza; hamburger; hot chips; potato, corn chips, etc.), cakes and biscuits (cakes, sweet muffins, scones or pikelets; sweet pies or sweet pastries; other puddings or desserts; plain sweet biscuits; cream chocolate biscuits) and confectionery (chocolate; other confectionery).

Food group variety scores were derived by summing the number of foods regularly consumed within each group. Total food variety was the sum of variety scores from all food groups. The food variety groups were defined as: total variety (range $0-88$ ), fruit variety (range 0-8), vegetable variety (range $0-25$ ), dairy variety (range $0-10$ ), cereal variety (range 0-9), fish variety (range 0-4), meat variety (range 0-15), snacks variety (range 0-5) and spreads variety (range $0-5$ ). Since the confectionery food group consisted of only two items, it was combined with the cakes and biscuits food group into a sweets variety group (range 0-7).

\section{Education}

Post-school education qualification was used as a measure of education. This information had been elicited as part of the NHS. For cost and time reasons, the ABS had administered some sections of the survey - among them the section covering education - to half the sample only. Sub-sample selections had been made on a block basis. Post-school education qualification could take the following values: (0) not applicable, (i) higher degree, (ii) postgraduate diploma, (iii) bachelor, (iv) undergraduate diploma, (v) associate diploma, (vi) skilled vocational, (vii) basic vocational, (viii) inadequately described and (ix) no higher qualifications. Twenty-four responses where post-school education qualifications had been inadequately described and 5490 responses that were in the 'not applicable' category because information about post-school education had not been assessed were declared to be missing. Post-school education qualification was then re-coded into the following three groups: category (ix) into (1) no higher qualifications, categories (vi) and (vii) into (2) vocational training, and categories (i) to (v) into (3) university.

\section{Statistical analyses}

Analyses were conducted using SPSS version $11^{34}$. All analyses were conducted within each sex since sex differences in food consumption are well established ${ }^{6,9,22}$ and were evident during preliminary analyses of the data. The relationships of education with the consumption of individual foods were examined via contingency tables analyses within each sex (CROSSTABS procedure with chisquare statistics).

The relationship between age and education was also investigated to assess the potential confounding effect of age. For this purpose, the sample was split into three approximately equal-sized age groups: 18 to 34 years, 35 to 49 years and 50 years or more. The relationship between age and education was found to be weak, but significant (Spearman's rho $=-0.01, \quad P<0.001$, $n=5240$ ), indicating a slight tendency for older people to be less well educated. Contingency table analyses were then performed for men and women separately and stratified by age, examining the relationship between education and single food items for respondents from the three age groups. The association between educational level and reported consumption of individual foods by gender as well as age was measured using odds ratios. For this purpose, all contingency table analyses were performed as $2 \times 2$ cross-tabulations, with participants who had no further education being chosen as baseline 
against which vocationally trained participants, as well as participants with tertiary education, were compared. Pearson's chi-square was used to infer statistical significance of these associations.

Two-way analyses of variance were performed separately for men and women to test for interactions between age group and education on the computed food group scores.

\section{Results}

\section{Demographic characteristics of the sample}

As can be seen in Table 1, a comparable percentage of men and women were in each of the three age groups. For both genders, the 50 year and above age group was more strongly represented than the two younger age groups. Slightly more men than women held higher qualifications. Compared with data from the 1996 census of population and housing ${ }^{35}$, and according to information provided by the $\mathrm{ABS}^{36}$, the current sample appears to be representative of the general population in terms of gender, age and education.

\section{Association of education with the consumption of individual foods}

For contingency table analyses, only relationships that were significant at $P<0.05$, as well as showing $50 \%$ or more increased (or decreased) regular consumption of foods by participants in higher educational groups in comparison to participants without higher education, were considered to be sufficiently important to be discussed. Education was related to the reported consumption of many individual foods (Table 2). Among university-educated participants, both men and women reported more regular consumption of the following foods than respondents without post-school education: apple or pear, banana, grapes or berries, mango, orange, peach, broccoli, capsicum (red pepper), lettuce, mushrooms, onion or leeks, other beans or lentils, side salad with meal, soybeans, stir-fried vegetables, tomato, zucchini (courgette), liver, mixed dishes with chicken, turkey or duck, other seafood, cream or sour cream, yoghurt, dry biscuits, muesli, pasta, rice, wholemeal bread, oil and vinegar dressing, and pizza. University education was also linked to more regular consumption of celery or cucumber, baked or grilled fish, cottage cheese, milk on breakfast cereal and sweet pies or pastry for men, and melon and potato or corn chips for women. Men with university education consumed roast lamb considerably less regularly than men without higher education.

Vocationally trained men consumed only one food carrots - considerably more regularly than men without post-school education, while vocationally trained women reported appreciably more regular consumption than women without post-school education of mushrooms, soybeans, stir-fried vegetables, sweet corn, roast chicken, mixed dishes with chicken, rice, pasta, hamburger and pizza, as well as potato or corn chips. Overall, men and women from the two lower educational groups tended to consume many foods with lesser regularity than did men and women with university education.

\section{Associations of education and age with the consumption of individual foods}

Stratification of the analyses by age showed that the number of strong and significant associations between reported regular consumption and education was higher for the tertiary-educated than for the vocationally trained, and did not favour any of the age groups. While tertiary education was for the majority of foods related to more regular consumption of foods, some foods were consumed less regularly by tertiary-educated participants than by those without further education. As for vegetables, tertiary-educated men aged 50 years or more were less regular consumers of cauliflower, while 35- to 49-year-old women consumed peas less regularly than did women without further education. Negative relationships between education and regular consumption of foods occurred predominantly among meats, and were more prevalent among men than among women. While both tertiaryeducated men and women in the middle age group reported less regular consumption of roast beef than men and women in the same age group without further education, the remainder of the negative relationships between education and regular consumption of meats occurred only among tertiary-educated men in the two

Table 1 Sample demographic characteristics

\begin{tabular}{|c|c|c|c|c|c|c|c|c|c|}
\hline & & \multicolumn{2}{|c|}{$18-34$ years } & \multicolumn{2}{|c|}{$35-49$ years } & \multicolumn{2}{|c|}{$\begin{array}{l}50 \text { years or } \\
\text { more }\end{array}$} & \multicolumn{2}{|c|}{ All ages } \\
\hline & & $n$ & $\%$ & $n$ & $\%$ & $n$ & $\%$ & $n$ & $\%$ \\
\hline \multirow[t]{4}{*}{ Men } & No post-school education & 383 & 49.9 & 280 & 36.8 & 477 & 49.0 & 1140 & 45.6 \\
\hline & Vocational training & 207 & 27.0 & 225 & 29.6 & 301 & 30.9 & 733 & 29.3 \\
\hline & University & 178 & 23.2 & 255 & 33.6 & 195 & 20.0 & 628 & 25.1 \\
\hline & All education groups & 768 & 30.7 & 760 & 30.4 & 973 & 38.9 & 2501 & 100.0 \\
\hline \multirow[t]{4}{*}{ Women } & No post-school education & 479 & 52.1 & 412 & 53.0 & 749 & 72.0 & 1640 & 59.9 \\
\hline & Vocational training & 192 & 20.9 & 143 & 18.4 & 140 & 13.4 & 475 & 17.3 \\
\hline & University & 249 & 27.1 & 223 & 28.7 & 152 & 14.6 & 624 & 22.8 \\
\hline & All education groups & 920 & 33.6 & 778 & 28.4 & 1041 & 38.0 & 2739 & 100.0 \\
\hline
\end{tabular}


Table 2 Regular consumption (\%) of individual foods for men (M) and women (F) without post-school education overall and in three different age groups, and odds ratios for vocationally and university-educated men and women relative to those with no post-school education - Australian National Nutrition Survey $1995 \dagger$

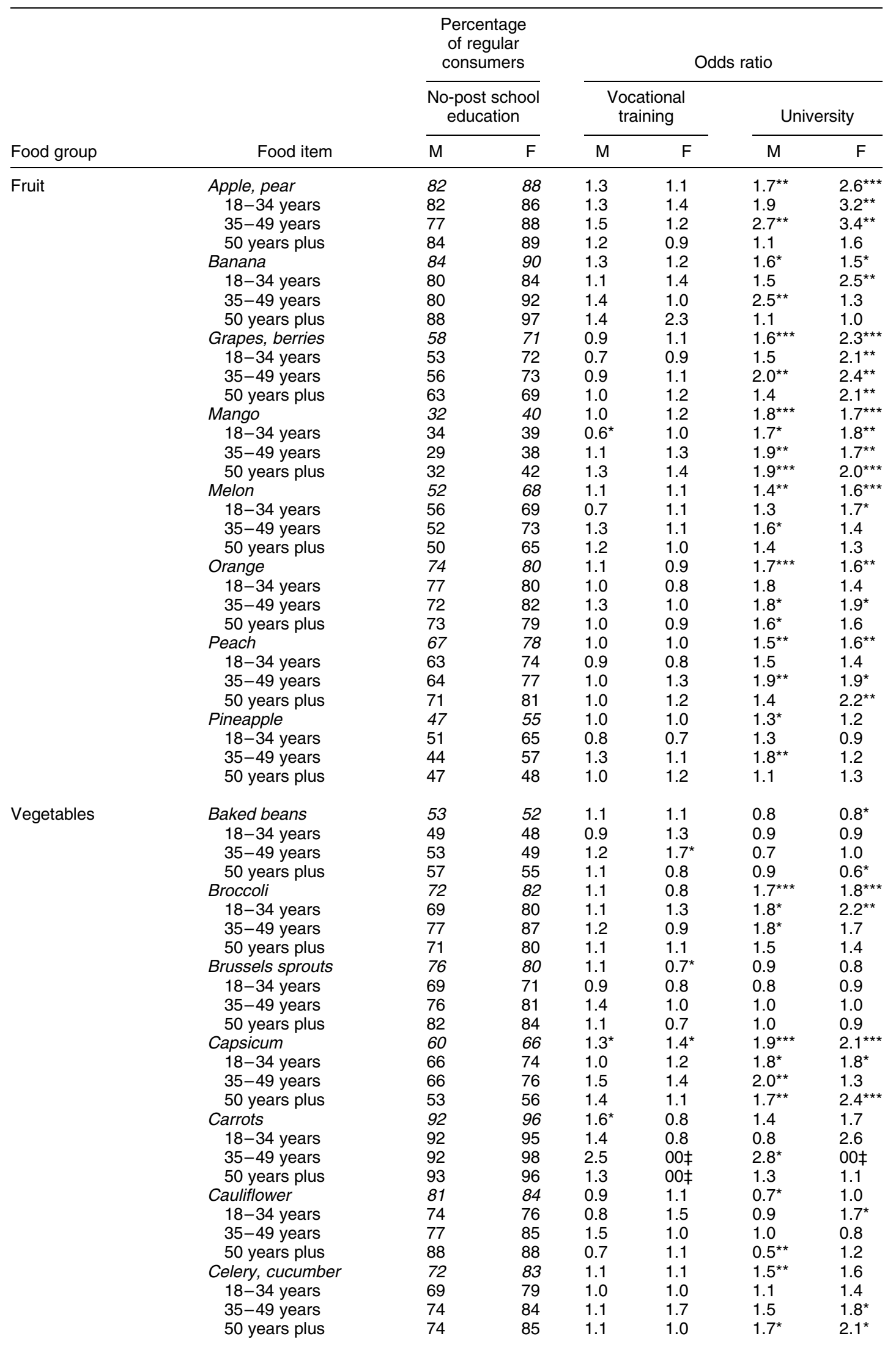


Table 2. Continued

\begin{tabular}{|c|c|c|c|c|c|c|c|}
\hline \multirow[b]{3}{*}{ Food group } & \multirow[b]{3}{*}{ Food item } & \multirow{2}{*}{\multicolumn{2}{|c|}{$\begin{array}{c}\begin{array}{c}\text { Percentage } \\
\text { of regular } \\
\text { consumers }\end{array} \\
\begin{array}{c}\text { No-post school } \\
\text { education }\end{array}\end{array}$}} & \multicolumn{4}{|c|}{ Odds ratio } \\
\hline & & & & \multicolumn{2}{|c|}{$\begin{array}{l}\text { Vocational } \\
\text { training }\end{array}$} & \multicolumn{2}{|c|}{ University } \\
\hline & & $\mathrm{M}$ & $\mathrm{F}$ & M & $\mathrm{F}$ & M & $\mathrm{F}$ \\
\hline & Green beans & 85 & 88 & 1.2 & 1.0 & 1.0 & 0.8 \\
\hline & $18-34$ years & 78 & 77 & 1.3 & 1.2 & 0.9 & 1.3 \\
\hline & $35-49$ years & 84 & 90 & 1.6 & 1.3 & 1.4 & 0.6 \\
\hline & 50 years plus & 92 & 93 & 0.7 & 1.1 & 0.7 & 1.5 \\
\hline & Lettuce & 88 & 92 & 0.9 & 1.0 & $1.8^{\star \star}$ & $1.6^{*}$ \\
\hline & $18-34$ years & 91 & 91 & 0.8 & 1.7 & 0.8 & 2.1 \\
\hline & $35-49$ years & 90 & 94 & 1.2 & 0.6 & $3.0^{*}$ & 1.2 \\
\hline & 50 years plus & 86 & 92 & 0.9 & 0.8 & $2.2^{*}$ & 1.4 \\
\hline & Mushrooms & 65 & 67 & 1.2 & $1.5^{\star \star}$ & $2.3^{\star \star \star}$ & $1.9^{\star \star *}$ \\
\hline & $18-34$ years & 67 & 69 & 1.2 & 1.5 & $2.4^{\star \star}$ & $1.9^{\star \star}$ \\
\hline & $35-49$ years & 70 & 70 & 1.4 & $2.0^{*}$ & $2.0^{\star \star}$ & $1.6^{\star}$ \\
\hline & 50 years plus & 61 & 64 & 1.2 & 1.1 & $2.4^{\star \star}$ & $2.0^{* * *}$ \\
\hline & Onion or leeks & 88 & 88 & 1.3 & 1.4 & $1.5^{\star}$ & $2.2^{\star \star \star}$ \\
\hline & $18-34$ years & 86 & 87 & 1.1 & 1.5 & 1.1 & $2.4^{\star}$ \\
\hline & $35-49$ years & 88 & 91 & 1.7 & 1.2 & $2.3^{*}$ & 2.2 \\
\hline & 50 years plus & 88 & 87 & 1.1 & 1.5 & 1.2 & 1.8 \\
\hline & Other beans, lentils & 28 & 31 & 0.9 & 0.9 & $1.8^{\star \star \star}$ & $1.6^{\star \star \star}$ \\
\hline & $18-34$ years & 26 & 29 & $0.4^{\star \star}$ & 0.7 & $2.0^{\star \star}$ & $1.8^{\star \star}$ \\
\hline & $35-49$ years & 27 & 32 & 1.4 & 0.9 & $1.8^{\star \star}$ & $1.6^{\star}$ \\
\hline & 50 years plus & 30 & 32 & 0.9 & 1.1 & $1.9^{\star \star}$ & 1.3 \\
\hline & Peas & 91 & 89 & 1.2 & 1.0 & $0.6^{\star \star}$ & $0.7^{\star \star}$ \\
\hline & $18-34$ years & 87 & 85 & 0.9 & 1.5 & 0.6 & 0.9 \\
\hline & $35-49$ years & 89 & 91 & 1.9 & 0.9 & 0.7 & $0.4^{\star *}$ \\
\hline & 50 years plus & 94 & 90 & 0.9 & 0.9 & 0.6 & 1.2 \\
\hline & Potato & 96 & 97 & 1.4 & 1.1 & 0.8 & 0.6 \\
\hline & $18-34$ years & 95 & 96 & 1.0 & $00 \ddagger$ & 0.6 & 0.5 \\
\hline & $35-49$ years & 95 & 97 & 2.9 & $00 \ddagger$ & 1.9 & 0.7 \\
\hline & 50 years plus & 98 & 98 & $00 \ddagger$ & $00 \ddagger$ & $00 \ddagger$ & $00 \ddagger$ \\
\hline & Pumpkin & 80 & 87 & 1.2 & 0.9 & 0.8 & 0.9 \\
\hline & $18-34$ years & 73 & 80 & 1.1 & 1.5 & 0.9 & 1.1 \\
\hline & $35-49$ years & 78 & 89 & 1.7 & 1.0 & 1.0 & 0.8 \\
\hline & 50 years plus & 85 & 90 & 0.9 & 0.6 & $0.6^{*}$ & 0.9 \\
\hline & Side salad with meal & 85 & 88 & 1.1 & 1.2 & $2.1^{\star \star \star}$ & $2.4^{\star \star \star}$ \\
\hline & $18-34$ years & 87 & 89 & 0.9 & 1.3 & 1.5 & $3.8^{\star \star}$ \\
\hline & $35-49$ years & 88 & 94 & 2.0 & 0.9 & $3.3^{\star \star}$ & 1.6 \\
\hline & 50 years plus & 81 & 84 & 1.0 & 1.0 & $1.9^{*}$ & 1.6 \\
\hline & Silverbeet, spinach & 49 & 56 & 1.0 & 0.8 & 1.2 & $1.3^{*}$ \\
\hline & $18-34$ years & 39 & 40 & 1.0 & 1.0 & 1.1 & $1.8^{\star \star}$ \\
\hline & $35-49$ years & 48 & 54 & 0.9 & 1.1 & 1.1 & $1.6^{*}$ \\
\hline & 50 years plus & 55 & 65 & 1.1 & 0.8 & 1.3 & 1.1 \\
\hline & Soybeans & 6 & 5 & 0.8 & $1.8^{\star \star}$ & $2.2^{\star \star \star}$ & $3.3^{\star \star \star}$ \\
\hline & $18-34$ years & 10 & 9 & 0.7 & 1.0 & 1.8 & 1.8 \\
\hline & $35-49$ years & 6 & 6 & 1.0 & $2.2^{*}$ & $2.4^{*}$ & $4.0^{\star \star \star}$ \\
\hline & 50 years plus & 4 & 4 & 1.0 & $00 \ddagger$ & $2.6^{*}$ & $3.9^{\star \star \star}$ \\
\hline & $\begin{array}{l}\text { Stir-fried or mixed } \\
\text { vegetables }\end{array}$ & 74 & 76 & 1.1 & $1.9^{* * *}$ & $1.9^{* * \star}$ & $2.6^{\star \star *}$ \\
\hline & $18-34$ years & 85 & 85 & 0.7 & 1.6 & 1.3 & 1.8 \\
\hline & $35-49$ years & 77 & 86 & 1.7 & 1.5 & $2.4^{\star \star}$ & $2.2^{*}$ \\
\hline & 50 years plus & 65 & 66 & 1.2 & $1.6^{*}$ & $1.8^{\star \star}$ & $2.2^{* *}$ \\
\hline & Sweet corn & 63 & 65 & 1.1 & $1.5^{\star \star}$ & 1.2 & $1.4^{\star \star}$ \\
\hline & $18-34$ years & 78 & 79 & 0.8 & 1.2 & $0.6^{*}$ & 1.0 \\
\hline & $35-49$ years & 64 & 74 & $1.6^{\star}$ & 1.4 & 1.5 & 0.8 \\
\hline & 50 years plus & 51 & 53 & 1.1 & 1.3 & 1.3 & $1.6^{*}$ \\
\hline & Sweet potato & 26 & 32 & 1.1 & 1.2 & 1.2 & $1.4^{\star \star}$ \\
\hline & $18-34$ years & 29 & 27 & 0.8 & 1.3 & 1.5 & 1.4 \\
\hline & $35-49$ years & 28 & 32 & 1.2 & 1.1 & 0.9 & 1.4 \\
\hline & 50 years plus & 23 & 33 & 1.2 & 1.3 & 1.2 & 1.5 \\
\hline & Tomato & 92 & 94 & 1.4 & 0.7 & $1.7^{*}$ & $2.0^{*}$ \\
\hline & $18-34$ years & 91 & 92 & 1.3 & 0.6 & 1.3 & $3.4^{\star \star}$ \\
\hline & $35-49$ years & 95 & 97 & 1.6 & $00 \ddagger$ & 2.1 & $00 \ddagger$ \\
\hline & 50 years plus & 92 & 94 & 1.4 & 0.8 & 1.7 & 0.9 \\
\hline & Vegetable casserole & 47 & 47 & 0.9 & $1.4^{\star *}$ & 1.2 & $1.3^{*}$ \\
\hline & $18-34$ years & 47 & 47 & 1.0 & $1.6^{*}$ & 1.2 & 1.3 \\
\hline
\end{tabular}




\begin{tabular}{|c|c|c|c|c|c|c|c|}
\hline \multirow[b]{3}{*}{ Food group } & \multirow[b]{3}{*}{ Food item } & \multirow{2}{*}{\multicolumn{2}{|c|}{$\begin{array}{c}\begin{array}{c}\text { Percentage } \\
\text { of regular } \\
\text { consumers }\end{array} \\
\begin{array}{c}\text { No-post school } \\
\text { education }\end{array}\end{array}$}} & \multicolumn{4}{|c|}{ Odds ratio } \\
\hline & & & & \multicolumn{2}{|c|}{$\begin{array}{c}\text { Vocational } \\
\text { training }\end{array}$} & \multicolumn{2}{|c|}{ University } \\
\hline & & $\mathrm{M}$ & $\mathrm{F}$ & $M$ & $\mathrm{~F}$ & $M$ & $\mathrm{~F}$ \\
\hline & $35-49$ years & 55 & 54 & 0.9 & 1.4 & 1.0 & 1.1 \\
\hline & 50 years plus & 42 & 43 & 0.9 & 1.1 & 1.2 & 1.1 \\
\hline & Vegetable sandwich & 84 & 89 & 0.9 & 1.0 & 1.2 & 1.0 \\
\hline & $18-34$ years & 88 & 90 & 0.8 & 1.1 & 1.6 & 1.1 \\
\hline & $35-49$ years & 88 & 91 & 1.3 & 1.4 & 1.6 & 0.7 \\
\hline & 50 years plus & 79 & 87 & 0.8 & 0.7 & 0.8 & 0.9 \\
\hline & Zucchini & 42 & 56 & 1.2 & 1.2 & $2.3^{\star \star \star}$ & $2.2^{\star \star \star}$ \\
\hline & $18-34$ years & 45 & 57 & 0.8 & 1.1 & $2.1^{\star \star}$ & $2.1^{\star \star \star}$ \\
\hline & $35-49$ years & 43 & 62 & 1.3 & 1.3 & $2.6^{\star \star *}$ & $1.7^{\star \star}$ \\
\hline & 50 years plus & 41 & 51 & 1.3 & 0.9 & $2.1^{\star \star \star}$ & $2.2^{\star \star \star}$ \\
\hline \multirow[t]{51}{*}{ Meat } & Bacon & 68 & 59 & 0.9 & 1.0 & 0.9 & 0.9 \\
\hline & $18-34$ years & 73 & 68 & 1.2 & 0.8 & 0.6 & 0.7 \\
\hline & $35-49$ years & 67 & 64 & 1.1 & 1.1 & 1.0 & $0.6^{*}$ \\
\hline & 50 years plus & 64 & 53 & 0.8 & 0.8 & 0.9 & 1.0 \\
\hline & Beef, veal & 93 & 90 & 0.9 & 0.7 & $0.7^{*}$ & $0.6^{* \star}$ \\
\hline & $18-34$ years & 94 & 87 & 1.1 & 0.8 & 0.6 & 0.8 \\
\hline & $35-49$ years & 95 & 90 & 0.7 & 0.8 & $0.4^{\star}$ & $0.5^{\star}$ \\
\hline & 50 years plus & 91 & 90 & 1.0 & 0.7 & 1.0 & 0.7 \\
\hline & Chicken, roast & 82 & 79 & 0.8 & $1.5^{\star}$ & 1.1 & 1.3 \\
\hline & $18-34$ years & 86 & 81 & 0.7 & 1.2 & 1.1 & 1.1 \\
\hline & $35-49$ years & 84 & 84 & 1.0 & 1.4 & 1.0 & 1.0 \\
\hline & 50 years plus & 79 & 76 & 0.9 & $1.7^{*}$ & 1.2 & 1.6 \\
\hline & $\mathrm{Ham}$ & 75 & 73 & 0.8 & 1.0 & 1.1 & 0.8 \\
\hline & $18-34$ years & 80 & 75 & 1.0 & 1.1 & 0.8 & 0.9 \\
\hline & $35-49$ years & 75 & 75 & 0.9 & 1.0 & 1.0 & 0.8 \\
\hline & 50 years plus & 72 & 71 & 0.8 & 0.7 & 1.5 & 0.7 \\
\hline & Lamb, roast & 83 & 81 & 0.8 & $0.7^{\star \star}$ & $0.5^{\star \star \star}$ & $0.6^{\star \star \star}$ \\
\hline & $18-34$ years & 83 & 78 & 0.9 & 0.7 & $0.5^{\star \star}$ & $0.6^{\star}$ \\
\hline & $35-49$ years & 82 & 78 & 0.9 & 1.0 & $0.4^{\star *}$ & $0.6^{*}$ \\
\hline & 50 years plus & 84 & 83 & 0.7 & $0.5^{\star \star}$ & $0.6^{\star}$ & 0.8 \\
\hline & Liver & 13 & 14 & 1.0 & 1.1 & $1.6^{\star \star}$ & $1.7^{\star \star \star}$ \\
\hline & $18-34$ years & 12 & 10 & $0.4^{\star}$ & 1.1 & $1.8^{\star}$ & $2.6^{\star \star \star}$ \\
\hline & $35-49$ years & 14 & 19 & 0.9 & 0.9 & 1.3 & 1.2 \\
\hline & 50 years plus & 13 & 14 & 1.4 & 1.3 & $1.7^{\star}$ & $1.6^{\star}$ \\
\hline & Luncheon meats & 50 & 39 & 1.1 & 1.2 & 0.9 & 1.2 \\
\hline & $18-34$ years & 66 & 54 & 1.0 & 0.9 & $0.6^{*}$ & 0.9 \\
\hline & $35-49$ years & 50 & 44 & 1.5 & 1.0 & 1.2 & 0.9 \\
\hline & 50 years plus & 38 & 28 & 1.2 & 1.2 & 0.9 & 1.1 \\
\hline & Mince dishes & 84 & 80 & $0.7^{\star \star}$ & 0.9 & $0.7^{\star \star}$ & 0.8 \\
\hline & $18-34$ years & 90 & 84 & $0.5^{\star}$ & 1.0 & 0.6 & 0.6 \\
\hline & $35-49$ years & 86 & 81 & 0.9 & 1.0 & $0.5^{\star}$ & 0.8 \\
\hline & 50 years plus & 79 & 78 & 0.7 & 0.8 & 0.8 & 0.8 \\
\hline & Mixed dishes with beef & 83 & 81 & 0.9 & 1.2 & 1.2 & $1.4^{\star}$ \\
\hline & $18-34$ years & 88 & 81 & 0.7 & 1.4 & 0.9 & 1.3 \\
\hline & $35-49$ years & 86 & 84 & 0.9 & 1.3 & 1.0 & 1.0 \\
\hline & 50 years plus & 78 & 78 & 1.0 & 1.0 & 1.4 & $1.8^{*}$ \\
\hline & $\begin{array}{l}\text { Mixed dishes with } \\
\text { chicken }\end{array}$ & 60 & 63 & 1.2 & $1.5^{\star \star}$ & $1.7^{\star \star \star}$ & $1.8^{\star \star \star}$ \\
\hline & $18-34$ years & 74 & 73 & 1.0 & $1.7^{*}$ & 1.4 & $1.9^{\star \star}$ \\
\hline & $35-49$ years & 60 & 71 & 1.5 & 1.0 & $2.1^{\star *}$ & 1.0 \\
\hline & 50 years plus & 51 & 55 & 1.2 & 1.2 & $1.7^{\star \star}$ & $1.9^{\star \star}$ \\
\hline & Mixed dishes with lamb & 64 & 61 & 0.9 & 1.0 & 1.0 & 0.9 \\
\hline & $18-34$ years & 74 & 65 & 0.7 & 0.9 & 0.7 & 0.8 \\
\hline & $35-49$ years & 64 & 64 & 1.0 & 1.2 & 1.1 & 0.7 \\
\hline & 50 years plus & 56 & 57 & 1.1 & 0.8 & 1.0 & 0.9 \\
\hline & Mixed dishes with pork & 46 & 40 & 0.9 & 0.8 & $0.6^{\star \star \star}$ & $0.7^{\star \star}$ \\
\hline & $18-34$ years & 54 & 43 & 0.9 & 1.0 & 0.8 & 0.9 \\
\hline & $35-49$ years & 41 & 42 & 1.4 & 1.1 & 1.4 & 1.0 \\
\hline & 50 years plus & 43 & 37 & 1.1 & 0.8 & 1.0 & 1.3 \\
\hline & Other offal & 26 & 25 & 1.1 & 1.2 & $1.3^{*}$ & $1.4^{\star \star}$ \\
\hline & $18-34$ years & 30 & 26 & 0.9 & 1.4 & 1.2 & $1.7^{\star \star}$ \\
\hline & $35-49$ years & 27 & 28 & 1.1 & 0.7 & 1.3 & 1.0 \\
\hline
\end{tabular}


Table 2. Continued

\begin{tabular}{|c|c|c|c|c|c|c|c|}
\hline \multirow[b]{3}{*}{ Food group } & \multirow[b]{3}{*}{ Food item } & \multirow{2}{*}{\multicolumn{2}{|c|}{$\begin{array}{c}\begin{array}{c}\text { Percentage } \\
\text { of regular } \\
\text { consumers }\end{array} \\
\begin{array}{c}\text { No-post school } \\
\text { education }\end{array}\end{array}$}} & \multicolumn{4}{|c|}{ Odds ratio } \\
\hline & & & & \multicolumn{2}{|c|}{$\begin{array}{c}\text { Vocational } \\
\text { training }\end{array}$} & \multicolumn{2}{|c|}{ University } \\
\hline & & M & $\mathrm{F}$ & $M$ & $F$ & $\mathrm{M}$ & $\mathrm{F}$ \\
\hline & 50 years plus & 23 & 22 & 1.1 & 1.4 & $1.5^{\star}$ & 1.2 \\
\hline & Pork, roast & 60 & 51 & 0.9 & 0.8 & $0.6^{\star \star \star}$ & $0.7^{\star *}$ \\
\hline & $18-34$ years & 61 & 47 & 0.8 & 0.8 & $0.5^{\star \star}$ & 0.8 \\
\hline & $35-49$ years & 56 & 53 & 1.0 & 1.2 & 0.8 & 0.7 \\
\hline & 50 years plus & 60 & 56 & 1.0 & $0.7^{\star}$ & 0.7 & 0.9 \\
\hline & Sausage & 75 & 60 & 0.8 & $0.8^{*}$ & $0.6^{\star \star \star}$ & $0.7^{\star *}$ \\
\hline & $18-34$ years & 80 & 67 & 1.1 & 0.7 & $0.4^{\star \star \star}$ & $0.6^{\star}$ \\
\hline & $35-49$ years & 77 & 61 & 0.8 & 0.8 & 0.6 & $0.6^{\star \star}$ \\
\hline & 50 years plus & 71 & 56 & 0.7 & $0.6^{*}$ & $0.6^{*}$ & 0.8 \\
\hline \multirow[t]{16}{*}{ Fish } & Fish, baked or grilled & 55 & 57 & 1.2 & $1.3^{*}$ & $1.8^{\star \star \star}$ & $1.4^{\star \star}$ \\
\hline & $18-34$ years & 55 & 46 & 1.2 & $1.5^{\star}$ & 1.5 & $2.0^{\star \star \star}$ \\
\hline & $35-49$ years & 51 & 55 & 1.4 & $1.6^{\star}$ & $2.0^{\star \star}$ & $1.5^{\star}$ \\
\hline & 50 years plus & 58 & 64 & 1.2 & 1.2 & $2.3^{\star \star \star}$ & 1.2 \\
\hline & Fish, canned & 55 & 64 & 1.2 & 1.0 & $1.4^{\star \star}$ & 1.1 \\
\hline & $18-34$ years & 52 & 59 & 1.2 & 0.9 & 1.3 & 1.2 \\
\hline & $35-49$ years & 53 & 63 & 1.3 & 1.2 & 1.4 & 1.1 \\
\hline & 50 years plus & 59 & 66 & 1.1 & 1.0 & $1.6^{\star}$ & 1.2 \\
\hline & Fish, fried & 55 & 43 & 0.9 & 1.0 & 0.9 & 1.0 \\
\hline & $18-34$ years & 53 & 41 & 0.9 & 0.9 & 0.9 & 0.8 \\
\hline & $35-49$ years & 58 & 49 & 1.1 & 1.0 & 1.0 & 0.9 \\
\hline & 50 years plus & 55 & 41 & 0.8 & 0.9 & 0.9 & 1.1 \\
\hline & Other seafood & 34 & 25 & 0.9 & $1.4^{\star \star}$ & $1.5^{\star \star}$ & $1.6^{\star * \star}$ \\
\hline & $18-34$ years & 47 & 31 & 0.8 & 0.9 & 0.9 & 1.5 \\
\hline & $35-49$ years & 41 & 30 & 0.7 & $1.7^{\star}$ & 1.3 & 1.3 \\
\hline & 50 years plus & 21 & 19 & 1.3 & 1.4 & $2.4^{\star \star \star}$ & 1.5 \\
\hline \multirow[t]{35}{*}{ Dairy } & Cheddar cheese & 92 & 92 & 0.9 & 1.2 & 1.1 & 1.2 \\
\hline & 18-34 years & 93 & 94 & 0.6 & 1.1 & 0.7 & 1.2 \\
\hline & $35-49$ years & 92 & 93 & 1.1 & 1.6 & 1.4 & 1.0 \\
\hline & 50 years plus & 91 & 91 & 1.0 & 0.9 & 1.1 & 1.0 \\
\hline & Cottage cheese & 13 & 23 & 0.9 & 1.0 & $1.8^{\star * \star}$ & $1.3^{*}$ \\
\hline & $18-34$ years & 14 & 21 & 0.7 & 0.9 & 1.5 & 1.5 \\
\hline & $35-49$ years & 13 & 23 & 0.8 & 1.3 & 1.7 & $1.5^{*}$ \\
\hline & 50 years plus & 23 & 13 & 1.1 & 1.0 & $2.0^{\star \star}$ & 1.0 \\
\hline & Cream, sour cream & 41 & 48 & 1.0 & 1.2 & $1.6^{\star * *}$ & $1.6^{\star \star \star}$ \\
\hline & $18-34$ years & 46 & 47 & 1.2 & 1.4 & $1.9^{\star \star}$ & $1.8^{\star \star}$ \\
\hline & $35-49$ years & 38 & 53 & 0.8 & 1.3 & $2.1^{\star * \star}$ & 1.3 \\
\hline & 50 years plus & 39 & 46 & 0.9 & 1.1 & 1.2 & $1.6^{\star}$ \\
\hline & Egg & 89 & 86 & 1.1 & 1.2 & 0.8 & 1.3 \\
\hline & $18-34$ years & 87 & 88 & 1.4 & 0.8 & 1.6 & 1.2 \\
\hline & $35-49$ years & 93 & 87 & 1.5 & 2.1 & 0.7 & 1.3 \\
\hline & 50 years plus & 89 & 84 & 0.8 & 1.3 & $0.6^{*}$ & 1.2 \\
\hline & Flavoured milk & 46 & 38 & 1.0 & 1.1 & 1.1 & $1.4^{\star \star *}$ \\
\hline & $18-34$ years & 73 & 62 & 0.8 & 1.0 & 0.9 & 0.9 \\
\hline & $35-49$ years & 52 & 41 & 1.1 & 0.7 & 0.9 & 1.0 \\
\hline & 50 years plus & 24 & 25 & 1.2 & 0.9 & 1.3 & $1.5^{*}$ \\
\hline & Ice cream & 77 & 65 & 0.9 & 0.9 & 0.9 & 0.9 \\
\hline & $18-34$ years & 77 & 67 & 0.8 & 0.7 & 1.3 & 0.7 \\
\hline & $35-49$ years & 72 & 64 & 1.4 & 0.9 & 1.1 & 1.1 \\
\hline & 50 years plus & 80 & 65 & $0.7^{\star}$ & 1.0 & 0.7 & 0.9 \\
\hline & Milk as drink & 50 & 45 & 1.0 & 1.1 & 0.9 & 1.0 \\
\hline & $18-34$ years & 69 & 61 & 0.8 & 0.9 & 0.8 & 0.8 \\
\hline & $35-49$ years & 51 & 43 & 1.4 & 0.9 & 0.8 & $0.7^{\star}$ \\
\hline & 50 years plus & 37 & 37 & 1.0 & 1.2 & 0.9 & 1.0 \\
\hline & Milk in hot beverages & 83 & 85 & 1.1 & 0.8 & 1.0 & 1.1 \\
\hline & $18-34$ years & 85 & 88 & 0.8 & 0.9 & 1.1 & 0.9 \\
\hline & $35-49$ years & 87 & 87 & 0.8 & 0.8 & 0.7 & 0.8 \\
\hline & 50 years plus & 80 & 82 & $1.5^{\star}$ & 0.7 & 1.0 & 1.3 \\
\hline & $\begin{array}{l}\text { Milk on breakfast } \\
\text { cereal }\end{array}$ & 78 & 78 & 1.1 & 0.8 & $1.5^{\star}$ & 1.2 \\
\hline & $18-34$ years & 80 & 75 & 1.0 & 1.1 & 1.7 & 1.5 \\
\hline & $35-49$ years & 77 & 74 & 1.0 & 1.1 & 1.6 & 1.4 \\
\hline
\end{tabular}




\begin{tabular}{|c|c|c|c|c|c|c|c|}
\hline \multirow[b]{3}{*}{ Food group } & \multirow[b]{3}{*}{ Food item } & \multirow{2}{*}{\multicolumn{2}{|c|}{$\begin{array}{c}\begin{array}{c}\text { Percentage } \\
\text { of regular } \\
\text { consumers }\end{array} \\
\begin{array}{c}\text { No-post school } \\
\text { education }\end{array}\end{array}$}} & \multicolumn{4}{|c|}{ Odds ratio } \\
\hline & & & & \multicolumn{2}{|c|}{$\begin{array}{c}\text { Vocational } \\
\text { training }\end{array}$} & \multicolumn{2}{|c|}{ University } \\
\hline & & $\mathrm{M}$ & $\mathrm{F}$ & M & $\mathrm{F}$ & $M$ & $\mathrm{~F}$ \\
\hline & 50 years plus & 77 & 82 & 1.3 & $0.6^{*}$ & 1.3 & 0.9 \\
\hline & Yoghurt & 32 & 53 & $1.3^{*}$ & 1.2 & $2.3^{\star \star \star}$ & $1.8^{\star \star \star}$ \\
\hline & $18-34$ years & 40 & 56 & 1.4 & 1.1 & $2.2^{\star \star \star}$ & $1.5^{\star}$ \\
\hline & $35-49$ years & 32 & 58 & 1.2 & 1.1 & $2.5^{\star \star \star}$ & $1.6^{\star}$ \\
\hline & 50 years plus & 25 & 50 & $1.4^{*}$ & 1.3 & $2.1^{* \star *}$ & $2.1^{\star \star \star}$ \\
\hline \multirow[t]{36}{*}{ Cereal } & Breakfast cereal & 76 & 75 & 0.9 & 1.0 & 1.2 & 1.0 \\
\hline & $18-34$ years & 79 & 77 & 1.1 & 0.9 & 1.7 & 1.0 \\
\hline & $35-49$ years & 74 & 70 & 1.0 & 1.4 & 1.6 & 1.2 \\
\hline & 50 years plus & 74 & 76 & 0.8 & 0.8 & 0.9 & 0.7 \\
\hline & Cooked porridge & 26 & 32 & $1.3^{*}$ & 1.1 & $1.3^{*}$ & 1.2 \\
\hline & $18-34$ years & 17 & 26 & 1.6 & 1.4 & $1.8^{*}$ & $1.5^{\star}$ \\
\hline & $35-49$ years & 24 & 24 & 1.0 & 1.3 & 1.2 & $1.6^{*}$ \\
\hline & 50 years plus & 33 & 39 & 1.3 & 1.1 & 1.2 & 1.1 \\
\hline & Dry biscuits & 70 & 79 & 0.9 & 1.2 & $1.8^{\star \star \star}$ & $1.6^{\star \star}$ \\
\hline & $18-34$ years & 69 & 77 & 0.8 & 1.4 & $1.7^{\star}$ & $1.7^{*}$ \\
\hline & $35-49$ years & 72 & 80 & 1.1 & 1.3 & $1.9^{\star \star}$ & 1.6 \\
\hline & 50 years plus & 71 & 80 & 0.8 & 1.0 & $1.7^{\star}$ & 1.4 \\
\hline & Muesli & 21 & 20 & 1.1 & 1.3 & $2.1^{\star \star \star}$ & $2.3^{\star \star \star}$ \\
\hline & $18-34$ years & 29 & 19 & 0.7 & 1.5 & 1.5 & $2.2^{\star \star \star}$ \\
\hline & $35-49$ years & 17 & 22 & $1.9^{*}$ & 1.0 & $2.7^{\star \star \star}$ & $2.1^{\star \star \star}$ \\
\hline & 50 years plus & 16 & 20 & 1.1 & 1.2 & $2.5^{\star \star \star}$ & $2.8^{\star \star \star}$ \\
\hline & Muffin & 35 & 38 & 1.0 & 1.0 & 1.1 & 1.2 \\
\hline & $18-34$ years & 46 & 49 & 0.7 & 0.8 & 1.1 & 0.9 \\
\hline & $35-49$ years & 35 & 41 & 1.1 & 0.7 & 1.4 & 1.3 \\
\hline & 50 years plus & 28 & 31 & 0.9 & $0.6^{*}$ & 1.0 & 0.9 \\
\hline & Rice & 80 & 82 & 0.9 & $1.6^{\star \star}$ & $2.7^{\star \star \star}$ & $3.3^{\star \star \star}$ \\
\hline & $18-34$ years & 88 & 88 & 0.6 & 1.2 & $2.6^{\star}$ & $3.8^{\star \star}$ \\
\hline & $35-49$ years & 79 & 84 & 1.2 & $2.7^{\star}$ & $4.2^{* \star *}$ & $3.2^{\star *}$ \\
\hline & 50 years plus & 75 & 79 & 1.1 & 1.3 & $2.1^{\star \star}$ & $2.2^{\star \star}$ \\
\hline & Pasta & 76 & 81 & 1.1 & $1.8^{\star *}$ & $2.0^{\star \star \star}$ & $2.4^{\star \star \star}$ \\
\hline & $18-34$ years & 93 & 96 & 0.7 & 1.5 & 1.7 & 1.7 \\
\hline & $35-49$ years & 81 & 90 & 1.3 & 2.0 & $2.6^{\star \star}$ & $2.5^{\star}$ \\
\hline & 50 years plus & 60 & 69 & 1.2 & 1.2 & $1.8^{* *}$ & 1.4 \\
\hline & White bread & 87 & 81 & 0.9 & 0.8 & 0.7 & 0.8 \\
\hline & $18-34$ years & 93 & 92 & 1.3 & 0.6 & 0.9 & $0.4^{\star *}$ \\
\hline & $35-49$ years & 92 & 86 & 0.6 & 0.6 & $0.4^{\star \star}$ & $0.5^{\star \star}$ \\
\hline & 50 years plus & 80 & 73 & 0.9 & 0.7 & 0.9 & 1.0 \\
\hline & Wholemeal bread & 68 & 78 & $1.3^{*}$ & 1.1 & $3.3^{\star \star \star}$ & $2.0^{\star \star \star}$ \\
\hline & $18-34$ years & 70 & 78 & 0.9 & 1.0 & $3.0^{\star \star \star}$ & 1.3 \\
\hline & $35-49$ years & 64 & 79 & 1.4 & 0.8 & $3.7^{\star \star \star}$ & $2.4^{\star \star}$ \\
\hline & 50 years plus & 70 & 79 & $1.5^{\star}$ & $1.9^{*}$ & $3.4^{\star \star \star}$ & $4.4^{\star \star \star}$ \\
\hline \multirow[t]{19}{*}{ Spreads } & Jam, marmalade & 76 & 74 & 1.1 & 0.9 & $1.4^{\star}$ & 1.1 \\
\hline & $18-34$ years & 70 & 66 & 1.3 & 1.0 & $2.6^{\star \star}$ & 1.4 \\
\hline & $35-49$ years & 70 & 73 & 1.0 & 1.2 & $1.6^{\star}$ & 1.5 \\
\hline & 50 years plus & 82 & 78 & 0.9 & 1.0 & 0.9 & 0.9 \\
\hline & Mayonnaise & 53 & 60 & 0.9 & 1.0 & 1.0 & 1.1 \\
\hline & $18-34$ years & 63 & 55 & 0.8 & 1.2 & 0.7 & 1.2 \\
\hline & $35-49$ years & 51 & 61 & 1.3 & 1.0 & 1.2 & 1.1 \\
\hline & 50 years plus & 48 & 62 & 0.9 & 0.8 & 1.1 & 1.0 \\
\hline & $\begin{array}{l}\text { Oil and vinegar } \\
\text { dressing }\end{array}$ & 46 & 51 & 1.1 & 1.1 & $1.9^{\star \star \star}$ & $1.8^{\star \star \star}$ \\
\hline & $18-34$ years & 48 & 49 & 0.9 & 1.1 & 1.4 & $1.7^{\star \star}$ \\
\hline & $35-49$ years & 50 & 56 & 1.1 & 1.4 & $1.7^{\star \star}$ & $1.6^{\star}$ \\
\hline & 50 years plus & 42 & 50 & 1.1 & 0.8 & $2.2^{\star \star \star}$ & $2.0^{\star \star \star}$ \\
\hline & Peanut butter & 42 & 45 & 1.1 & 1.1 & $1.4^{\star *}$ & $1.4^{\star \star}$ \\
\hline & $18-34$ years & 52 & 52 & 1.0 & 1.3 & 1.4 & 1.1 \\
\hline & $35-49$ years & 44 & 50 & 1.3 & 0.9 & 1.4 & 1.3 \\
\hline & 50 years plus & 34 & 40 & 1.2 & 1.0 & 1.3 & 1.4 \\
\hline & Vegemite & 62 & 67 & 1.0 & 1.0 & 0.9 & 1.1 \\
\hline & $18-34$ years & 74 & 76 & 1.0 & 1.0 & 0.7 & 1.0 \\
\hline & $35-49$ years & 65 & 72 & 1.0 & 0.9 & 0.8 & 0.7 \\
\hline
\end{tabular}


Table 2. Continued

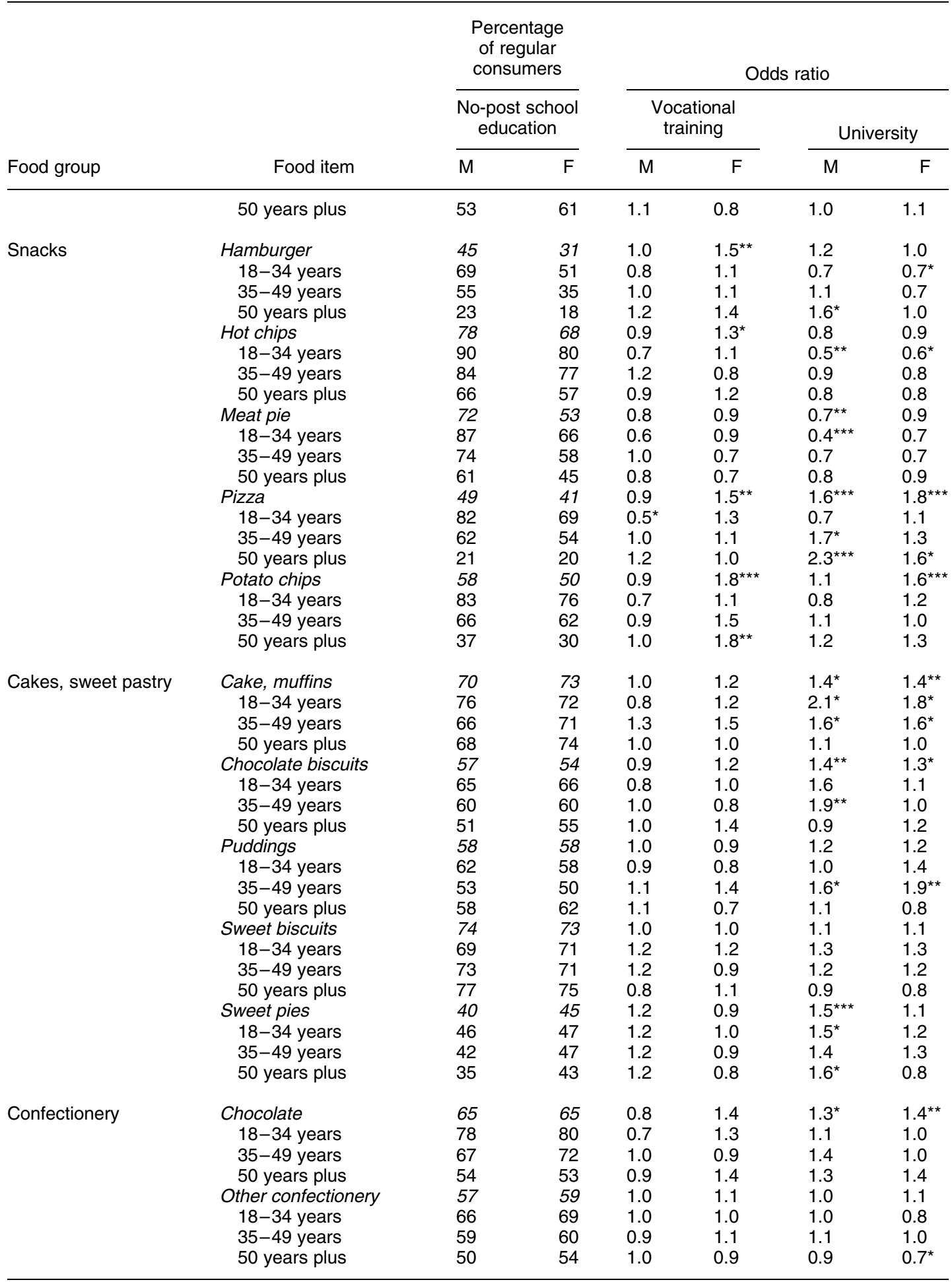

†For sample size, refer to Table 1.

$\ddagger$ No odds ratios are given due to the number of expected frequencies being less than 5 .

Asterisks denote significant departures in comparison to baseline level (i.e. no post-school education): ${ }^{*}, P<0.05 ;{ }^{\star *}, P<0.01 ;{ }^{* \star *}$,

$P<0.001$. 
younger age groups. Men aged 18 to 34 years reported less regular consumption of roast lamb, roast pork and sausage, while men aged 35 to 49 years consumed roast lamb and mince dishes less regularly than men in the same age group without further education. Tertiary-educated men and women aged 35 to 49 years were significantly less regular consumers of white bread than were participants in the same age group who had no further education, and so were tertiary-educated women aged 18 to 34 years. Tertiary-educated men aged 18 to 34 years also consumed hot chips and meat pie considerably less regularly than young men without further education.

Only comparatively few strong associations between education and the consumption of individual foods emerged for vocationally trained men and women in the three age groups. Relationships were approximately evenly distributed among the three age groups, but a higher number of strong and significant relationships occurred among women than among men. Similar to participants with university education, vocationally trained men or women in various age groups reported more regular consumption of mushrooms, soybeans, sweet corn, mixed dishes with chicken, baked fish, other seafood, muesli, rice and wholemeal bread relative to participants without further education, and less regular consumption of mince dishes. In addition, vocationally trained men aged 50 years or over reported more regular consumption of milk in hot beverages than same-aged men without further education, while women in the middle age group consumed baked beans more regularly and those aged 50 years or more reported a higher consumption of potato chips than comparatively aged women without further education. Against the trend was the reported consumption of other beans or lentils, liver and pizza. While more regular consumption of these foods had been reported by tertiary-educated men and women from various age groups, vocationally trained men aged 18 to 34 years consumed these foods with less regularity than young men without further education.

\section{Association of education with food variety scores}

Non university-educated men and women tended to consume a smaller total variety of foods than universityeducated people (Fig. 1). The relationship between education and food variety appeared to be less pronounced for men than for women, with men from the two lower educational groups showing no difference in food variety. Among women, however, the relationship was linear, and variety scores increased with education.

The variety of foods consumed from most of the food groups tended to increase with higher education (Table 3). University-educated men had significantly greater variety scores in most food groups than other men. The exceptions were meat and snacks, which exhibited no significant education differences. The only food group for which men with vocational qualifications differed

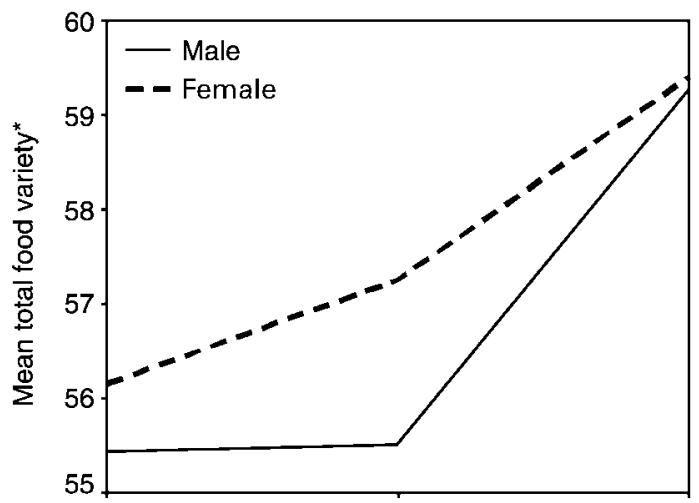

No higher education Vocational training University degree Highest educational qualification

Fig. 1 The relationship between education and mean total food variety for men and women (*average score of the sum of all dichotomous foods)

Table 3 Food group variety for men and women from three educational groups: mean and standard deviation (SD)

\begin{tabular}{|c|c|c|c|c|c|c|c|}
\hline & & $\begin{array}{l}\text { No } \\
\text { sch } \\
\text { educ }\end{array}$ & $\begin{array}{l}\text { ost- } \\
\text { ool } \\
\text { tion* }\end{array}$ & $\begin{array}{r}\text { Voc } \\
\text { tra }\end{array}$ & $\begin{array}{l}\text { nal } \\
g^{\star}\end{array}$ & Unive & sity* \\
\hline & Food group & Mean & SD & Mean & SD & Mean & SD \\
\hline Men & Cereal & $5.4^{\mathrm{a}}$ & 1.7 & $5.4^{\mathrm{a}}$ & 1.7 & $6.1^{\mathrm{b}}$ & 1.5 \\
\hline & Dairy & $6.0^{\mathrm{a}}$ & 1.8 & $6.0^{\mathrm{a}}$ & 1.8 & $6.4^{\mathrm{b}}$ & 1.9 \\
\hline & Fish & $2.0^{\mathrm{a}}$ & 1.2 & $2.1^{a}$ & 1.3 & $2.3^{\mathrm{b}}$ & 1.2 \\
\hline & Fruit & $5.0^{\mathrm{a}}$ & 2.3 & $5.0^{\mathrm{a}}$ & 2.2 & $5.6^{\mathrm{b}}$ & 2.1 \\
\hline & Meat & $9.6^{a}$ & 3.1 & $9.5^{\mathrm{a}}$ & 3.2 & $9.4^{a}$ & 3.4 \\
\hline & Snacks & $3.0^{a}$ & 1.6 & $2.9^{a}$ & 1.6 & $3.1^{a}$ & 1.6 \\
\hline & Spreads & $2.8^{a}$ & 1.4 & $2.8^{\mathrm{a}, \mathrm{b}}$ & 1.4 & $3.0^{\mathrm{b}}$ & 1.4 \\
\hline & Sweets & $4.2^{\mathrm{a}}$ & 2.2 & $4.2^{\mathrm{a}}$ & 2.2 & $4.6^{\mathrm{b}}$ & 2.1 \\
\hline & Vegetables & $16.9^{\mathrm{a}}$ & 4.4 & $17.4^{\mathrm{b}}$ & 4.1 & $18.1^{\mathrm{c}}$ & 4.2 \\
\hline & Total variety & $55.5^{\mathrm{a}}$ & 12.1 & $55.5^{\mathrm{a}}$ & 11.9 & $59.3^{\mathrm{b}}$ & 11.9 \\
\hline Women & Cereal & $5.6^{a}$ & 1.6 & $5.8^{\mathrm{a}}$ & 1.6 & $6.2^{\mathrm{b}}$ & 1.5 \\
\hline & Dairy & $6.1^{a}$ & 1.9 & $6.2^{\mathrm{a}}$ & 1.9 & $6.6^{\mathrm{b}}$ & 2.0 \\
\hline & Fish & $1.9^{a}$ & 1.2 & $2.0^{\mathrm{a}, \mathrm{b}}$ & 1.2 & $2.1^{\mathrm{b}}$ & 1.2 \\
\hline & Fruit & $5.7^{\mathrm{a}}$ & 2.0 & $5.8^{\mathrm{a}}$ & 2.1 & $6.4^{\mathrm{b}}$ & 1.7 \\
\hline & Meat & $8.9^{a}$ & 3.2 & $8.9^{\mathrm{a}}$ & 3.3 & $8.9^{\mathrm{a}}$ & 3.7 \\
\hline & Snacks & $2.4^{\mathrm{a}}$ & 1.6 & $2.8^{\mathrm{b}}$ & 1.7 & $2.7^{\mathrm{b}}$ & 1.5 \\
\hline & Spreads & $3.0^{a}$ & 1.3 & $3.0^{\mathrm{a}}$ & 1.4 & $3.2^{\mathrm{b}}$ & 1.3 \\
\hline & Sweets & $4.3^{\mathrm{a}}$ & 2.1 & $4.4^{\mathrm{a}}$ & 2.1 & $4.5^{\mathrm{a}}$ & 2.1 \\
\hline & Vegetak & $17.9^{\mathrm{a}}$ & 3.8 & $18.2^{\mathrm{a}}$ & 4.2 & $19.1^{\mathrm{b}}$ & 3.5 \\
\hline & Total variety & $56.2^{\mathrm{a}}$ & 11.5 & $57.3^{\mathrm{a}}$ & 11.8 & $59.4^{\mathrm{b}}$ & 11.0 \\
\hline
\end{tabular}

*Sample size: men $-n=1140,733$ and 628 for no post-school education, vocational training and university, respectively; women $-n=1640,475$ and 624 for no post-school education, vocational training and university, respectively.

For each measure, means that do not differ significantly at $P<0.017(\alpha / k$, where $k$ is the number of comparisons) share superscripts.

significantly from the other two educational groups was vegetables; this food group exhibited a positive linear relationship with education.

With the exception of meat and sweets, where no significant differences between groups emerged, women with higher education had significantly higher variety scores than women without higher education. Women with vocational training had, overall, comparable food variety scores to women without higher education. 
For snacks, however, women with vocational training were similar to university-educated women in consuming a significantly greater variety than women without higher education, while vocationally trained women did not differ significantly from either of the other educational groups in the variety of fish consumed.

\section{Association of age with food variety scores}

For most food groups, men aged 50 years or over reported significantly less variety than men aged 18 to 34 years (Table 4). The only exceptions to this pattern were fish, fruit and vegetables, with no significant differences in variety occurring between the three age groups.

Like the men, women aged 50 years or over also reported significantly lower scores on food group variety than women aged 18 to 34 years, although no age group differences were found with respect to fruit variety. Women aged 35 to 49 years had a significantly more varied intake of fish and vegetables than women aged 18 to 34 years.

\section{Associations of education and age with food variety scores}

Only snack variety for men showed a significant education by age group interaction $(F(4,1836)=2.97, P<0.05$, partial $\left.\eta^{2}=0.01\right)$. Men aged 18 to 34 years consumed, on average, a smaller variety of snacks the more educated they were, while the opposite pattern was found for men

Table 4 Food group variety for men and women from three age groups: mean and standard deviation (SD)

\begin{tabular}{|c|c|c|c|c|c|c|c|}
\hline & \multirow[b]{2}{*}{ Food group } & \multicolumn{2}{|c|}{$18-34$ years $^{*}$} & \multicolumn{2}{|c|}{$\begin{array}{l}35-49 \\
\text { years* }\end{array}$} & \multicolumn{2}{|c|}{$\begin{array}{l}50 \text { years } \\
\text { plus* }\end{array}$} \\
\hline & & Mean & SD & Mean & SD & Mean & SD \\
\hline Men & $\begin{array}{l}\text { Cereal } \\
\text { Dairy } \\
\text { Fish } \\
\text { Fruit } \\
\text { Meat } \\
\text { Snacks } \\
\text { Spreads } \\
\text { Sweets } \\
\text { Vegetables } \\
\text { Total variety }\end{array}$ & $\begin{array}{r}5.9^{\mathrm{a}} \\
6.8^{\mathrm{a}} \\
2.1^{\mathrm{a}} \\
5.0^{\mathrm{a}} \\
10.1^{\mathrm{a}} \\
3.9^{\mathrm{a}} \\
3.1^{\mathrm{a}} \\
4.7^{\mathrm{a}} \\
16.9^{\mathrm{a}} \\
58.3^{\mathrm{a}}\end{array}$ & $\begin{array}{r}1.6 \\
1.9 \\
1.4 \\
2.4 \\
3.1 \\
1.3 \\
1.4 \\
2.2 \\
4.8 \\
12.7\end{array}$ & $\begin{array}{r}5.7^{\mathrm{a}} \\
6.2^{\mathrm{b}} \\
2.2^{\mathrm{a}} \\
5.2^{\mathrm{a}} \\
9.6^{\mathrm{a}} \\
3.4^{\mathrm{b}} \\
2.9^{\mathrm{a}} \\
4.4^{\mathrm{a}} \\
18.1^{\mathrm{b}} \\
58.1^{\mathrm{a}}\end{array}$ & $\begin{array}{r}1.7 \\
1.8 \\
1.3 \\
2.3 \\
3.3 \\
1.4 \\
1.4 \\
2.2 \\
3.9 \\
11.9\end{array}$ & $\begin{array}{r}5.2^{\mathrm{b}} \\
5.5^{\mathrm{c}} \\
2.0^{\mathrm{a}} \\
5.2^{\mathrm{a}} \\
9.0^{\mathrm{b}} \\
2.1^{\mathrm{c}} \\
2.7^{\mathrm{b}} \\
4.0^{\mathrm{b}} \\
17.1^{\mathrm{a}} \\
53.6^{\mathrm{b}}\end{array}$ & $\begin{array}{r}1.6 \\
1.7 \\
1.1 \\
2.1 \\
3.1 \\
1.4 \\
1.4 \\
2.2 \\
4.1 \\
11.1\end{array}$ \\
\hline Women & $\begin{array}{l}\text { Cereal } \\
\text { Dairy } \\
\text { Fish } \\
\text { Fruit } \\
\text { Meat } \\
\text { Snacks } \\
\text { Spreads } \\
\text { Sweets } \\
\text { Vegetables } \\
\text { Total variety }\end{array}$ & $\begin{array}{l}6.1^{\mathrm{a}} \\
6.7^{\mathrm{a}} \\
1.9^{\mathrm{a}} \\
5.9^{\mathrm{a}} \\
9.3^{\mathrm{a}} \\
3.4^{\mathrm{a}} \\
3.1^{\mathrm{a}, \mathrm{b}} \\
4.7^{\mathrm{a}} \\
18.0^{\mathrm{a}} \\
59.1^{\mathrm{a}}\end{array}$ & $\begin{array}{c}1.5 \\
1.9 \\
1.3 \\
2.1 \\
3.4 \\
1.4 \\
1.3 \\
2.0 \\
4.06 \\
10.9\end{array}$ & $\begin{array}{c}5.9^{\mathrm{a}} \\
6.3^{\mathrm{b}} \\
2.1^{\mathrm{b}} \\
6.0^{\mathrm{a}} \\
9.1^{\mathrm{a}} \\
2.8^{\mathrm{b}} \\
3.2^{\mathrm{b}} \\
4.4^{\mathrm{a}} \\
19.0^{\mathrm{b}} \\
58.6^{\mathrm{a}}\end{array}$ & $\begin{array}{r}1.6 \\
2.0 \\
1.3 \\
1.9 \\
3.4 \\
1.5 \\
1.4 \\
2.1 \\
3.4 \\
11.6\end{array}$ & $\begin{array}{l}5.5^{\mathrm{b}} \\
5.9^{\mathrm{c}} \\
1.9^{\mathrm{a}, \mathrm{b}} \\
5.8^{\mathrm{a}} \\
8.5^{\mathrm{b}} \\
1.7^{\mathrm{c}} \\
2.9^{\mathrm{a}} \\
4.0^{\mathrm{b}} \\
17.9^{\mathrm{a}} \\
54.1^{\mathrm{b}}\end{array}$ & $\begin{array}{r}1.6 \\
1.8 \\
1.1 \\
1.9 \\
3.2 \\
1.4 \\
1.4 \\
2.1 \\
3.9 \\
11.4\end{array}$ \\
\hline
\end{tabular}

*Sample size: men $-n=774,760$ and 973 for age group 18-34 years, $35-49$ years and 50 years plus, respectively; women $-n=921,778$ and 1041 for age group 18-34 years, 35-49 years and 50 years plus, respectively.

For each measure, means that do not differ significantly at $P<0.017$ $(\alpha / k$, where $k$ is the number of comparisons) share superscripts. aged 50 years or more. The effect size of this interaction was, however, very small. There were no significant interactions between age group and education in the combined-sexes dataset.

\section{Discussion}

Educational background and age were both associated with the regularity of food consumption among men and women. Generally, university-educated people in all three age groups reported more regular consumption of foods that are regarded as 'novel' or 'healthy' (such as legumes, liver, whole-grain bread, yoghurt and certain fruits and vegetables) than their less-educated counterparts. These preferences were offset by reduced preferences for more traditional foods like roast meat, meat pie, sausages and white bread. Similar findings in Australia have been reported by Turrell et $a l .{ }^{27}$ In this respect, our findings are supportive of previous studies ${ }^{17}$ and social researchers ${ }^{16}$ who suggest that 'new knowledge' is first acquired and put into practice by the highly educated whilst the lesseducated cling to more traditional ways. With respect to some foods, the vocationally educated tended to occupy a middle position between those without further education and the university-educated group.

These observations raise questions about the nature of the effects of higher education and the nature of the 'healthy/novel' foods. A cross-sectional survey such as the NNS cannot establish any causal relationships between education and the types of food consumed. However, the findings do show that the dietary patterns of universityeducated people differ from those of less-educated people. Since higher educational attainment is linked to higher average earnings in OECD (Organization for Economic Co-operation and Development) countries ${ }^{37}$, it might be expected that the tertiary educated may be more able to purchase less common foods such as seafood other than fish, mango, or lean meats. In line with previous research ${ }^{12}$, however, the findings of the current study indicate that some of the foods preferred by the tertiaryeducated - such as broccoli, rice or liver - are relatively inexpensive. This suggests that income per se is not a key predictor of food consumption, but raises the possibility that, as sociologists like Bourdieu ${ }^{38}$ argue, food consumption patterns are a means of expressing one's social identity. The availability of economic capital together with the identification with a social class may thus determine food choices. Alternatively, self-selection factors may operate (i.e. people with certain dietary propensities go to university more often), or a process of change that is initiated by, or related to, higher education, resulting in the choice of different kinds of food by people who have undergone further education. Both these latter processes may operate simultaneously.

The resolution of these questions is a matter for future longitudinal research. However, recent studies suggest 
that higher education may involve a process of change in culture that people adopt when they start on the path to higher education. For example, Marmot and colleagues ${ }^{17}$ have shown that differences in socio-economic status (based in part on educational background) may be largely accounted for by position in the 'social hierarchy' and associated perceptions of powerlessness. People who are less educated often feel less able to influence the outcome of significant events in their lives. In a series of studies on attitudes towards dental care, Davies ${ }^{18}$ has shown that university education is strongly associated with a belief system that views disease outcomes as being under personal control rather than being 'inevitable'. Thus university-educated people may choose 'healthy' foods because they believe that their health is under their control, and this is one means by which they can improve or maintain good health.

However, this assumption about the healthier quality of the food choices made by those with tertiary education may be more apparent than real. Most of the foods shown in Table 2 which are more regularly consumed by the higher educated (such as grapes, capsicum, yoghurt, rice or wholemeal bread) may seem to be 'healthy' in terms of currently recommended dietary guidelines. But this group also includes cream, savoury biscuits, and sweet pies and pastry for men and potato or corn chips for women - all of which are major sources of saturated fat. So whilst the majority of tertiary-preferred foods may be considered 'healthy', quite a few are not recommended by the dietary guidelines $^{39}$.

A related theme may be tradition. Some of the foods preferred by the non university-educated are highly traditional, such as pies, peas and sausages. Tertiary- and vocationally educated men, in particular, eschewed several traditional red meats. This preference for novelty has been reported previously ${ }^{40}$. An instructive example is yoghurt, which is on the tertiary-preferred list. This food was introduced into Australia as a slimming food and has been only lately promoted as a health (lactobacilluscontaining) food.

Broadly speaking, the analyses of food group variety scores paralleled the analyses of individual food associations, but there were some notable differences. First, there was a general absence of significant education by age group interactions on the group variety scores (except for snacks among men). Second, there were significant education associations for several meats but no significant education group differences in meat variety scores. Thus, aggregation of the consumption data into variety scores may have reduced the pronounced differences seen among educational groups in the consumption of the individual foods.

Inspection of the variety scores of men and women confirmed that non university-educated people tended to consume less varied diets than the tertiary-educated. For both men and women, food variety scores of the high- school-educated and vocationally trained were similar for most food groups. This suggests that university education, rather than vocational education, is important in terms of food choices.

The variety scores for meats, snacks (for men) and sweets (for women) were unrelated to education. In part this may be due to aggregation effects, particularly with regard to meat consumption. It also suggests, however, that factors other than 'healthiness' and novelty may influence the consumption of these food groups. For example, Benton ${ }^{41}$ in his review of mood and food relationships has observed that high carbohydrate/sugar foods like snacks and sweets are widely used to improve poor mood states.

The sole interaction of age and education on men's snack variety scores may be related to tertiary-educated young men's greater involvement in long hours of study or employment, which may reduce their access to a wide variety of snacks. In contrast, the greater consumption of snacks by tertiary-educated men over 50 years may be linked to reduced time pressures and perhaps to the availability of partners who may prepare snacks for them. Clearly further research is needed.

The large sample size and its national representativeness are among the strengths of this study. The FFQ offers the advantage of assessing respondents' habitual consumption of individual foods or groups of food over an extended period of time, and it is thus less susceptible than short-term measures to be severely affected by temporary dietary change or by underreporting of specific food intakes. A major shortcoming of the FFQ, however, lies in the demand it makes on the cognitive processes of users in not only requiring a high literacy level and adequate longterm memory, but also the ability to average food intake over 12 months ${ }^{42}$. None the less, the consistency between current findings and those of past studies using various measures and showing education group differences in food consumption lend confidence to the results. Considering that people in the middle range of both age and income were more likely to participate in this survey than people at the extreme of these distributions, the results of this study may underestimate the strength of the relationship between education and the regular consumption of foods. The reported findings highlight the need to educate children about healthy food choices before they leave the school system.

\section{Conclusions}

1. Higher education is associated with the regular consumption of a wider variety of foods.

2. Aggregation of individual food consumption indices into food variety scores may reduce the apparent effects of educational background on food consumption. 


\section{Acknowledgements}

K.B. is supported by a Public Health Postdoctoral Research Fellowship from the National Health and Medical Research Council (NHMRC), ID 136925. D.C. is supported by a Career Development Award funded by the NHMRC and the National Heart Foundation of Australia. We are grateful to Associate Professor Damien Jolley for advice on statistical analyses and interpretation, and to an anonymous reviewer of this paper for suggested changes.

\section{References}

1 Benjamin-Garner R, Oakes M, Meischke H, Meshack A Stone EJ, Zapka J, et al. Sociodemographic differences in exposure to health information. Ethnicity \& Disease 2002; 12: $124-34$.

2 Finnegan JR Jr, Viswanath K, Rooney B, McGovern P, Baxter J, Elmer $\mathrm{P}$, et al. Predictors of knowledge about healthy eating in a rural midwestern US city. Health Education Research 1990; 5: 421-31.

3 Schafer RB, Schafer E, Dunbar M, Keith PM. Marital food interaction and dietary behavior. Social Science \& Medicine 1999; 48: 787-96.

4 Kline RL, Terry RD. Differences in beliefs about heart disease risk factors between men and women. Journal of the American Dietetic Association 1986; 86: 786-8.

5 Schafer RB. Factors affecting food behavior and the quality of husbands' and wives' diets. Journal of the American Dietetic Association 1978; 72: 138-43.

6 Fagerli RA, Wandel M. Gender differences in opinions and practices with regard to a 'healthy diet'. Appetite 1999; 32: 171-90.

7 Smith SC, Taylor JG, Stephen AM. Use of food labels and beliefs about diet-disease relationships among university students. Public Health Nutrition 2000; 3: 175-82.

8 Shannon C, Story M, Fulkerson JA, French SA. Factors in the school cafeteria influencing food choices by high school students. Journal of School Health 2002; 72: 229-35.

9 Milligan RAK, Burke V, Beilin LJ, Dunbar DL, Spencer MJ, Balde E, et al. Influence of gender and socio-economic status on dietary patterns and nutrient intakes in 18-year-old Australians. Australian and New Zealand Journal of Public Health 1998; 22: 485-93.

10 Bermudez OI, Dwyer J. Identifying elders at risk of malnutrition: a universal challenge. SCN News 1999; 19: $15-7$.

11 Horwath CC, Worsley A. Dietary supplement use in a randomly selected group of elderly Australians. Results from a large nutrition and health survey. Journal of the American Geriatrics Society 1989; 37: 689-96.

12 Worsley A, Blasche R, Ball K, Crawford D. Income differences in food consumption in the 1995 Australian Nutrition Survey. European Journal of Clinical Nutrition 2003; 57: 1198-211.

13 Wardle J, Parmenter K, Waller J. Nutrition knowledge and food intake. Appetite 2000; 34: 269-75.

14 Galobardes B, Morabia A, Bernstein MS. Diet and socioeconomic position: does the use of different indicators matter? International Journal of Epidemiology 2001; 30: 334-40.

15 Hupkens CLH, Knibbe RA, Drop MJ. Social class differences in food consumption. European Journal of Clinical Nutrition 2000; 10: 108-13.

16 Rogers EM. Diffusion of Innovations, 4th ed. New York: Free Press, 1995.
17 Marmot M, Wilkinson R, eds. Social Determinants of Health. Oxford: Oxford University Press, 1999.

18 Davies M. The role of commonsense understandings in social inequalities in bealth: an investigation in the context of dental bealth. PhD dissertation, Faculty of Medicine, University of Adelaide, Australia, 2000.

19 Ippolito RA. Education versus Savings as Explanation for Better Health: Evidence from the Health and Retirement Survey. Working Paper in Law and Economics No. 03-04. Arlington, VA: School of Law, George Mason University, 2003.

20 McKernan Boulanger P, Perez-Escamilla R, Himmelgreen D, Segura-Millan S, Haldeman L. Determinants of nutrition knowledge among low-income Latino caretakers in Hartford, Conn. Journal of the American Dietetic Association 2002; 7: 978-81.

21 Johansson L, Thelle DS, Solvoll K, Bjørneboe G-E, Drevon CA. Healthy dietary habits in relation to social determinants and lifestyle factors. British Journal of Nutrition 1999; $\mathbf{8 1}$ : 211-20.

22 Kearney M, Kearney J, Dunne A, Gibney M. Sociodemographic determinants of perceived influences on food choice in a nationally representative sample of Irish adults. Public Health Nutrition 2000; 3: 219-26.

23 Roos G, Johansson L, Kasmel A, Klumbiene J, Prättälä R. Disparities in vegetable and fruit consumption: European cases from the north to the south. Public Health Nutrition 2001; 4: 35-43.

24 Erkkilla AT, Sarkkinen ES, Lehto S, Pyorala K, Uusitupa ML. Diet in relation to socioeconomic status in patients with coronary heart disease. European Journal of Clinical Nutrition 1999; 53: 662-8.

25 Baghurst K, Record S, Baghurst P, Syrette J, Crawford D, Worsley A. Socioeconomic determinants in Australia of the intake of food and nutrients implicated in cancer aetiology. Medical Journal of Australia 1990; 153: 444-52.

26 Smith A, Owen N. Associations of social status and healthrelated beliefs with dietary fat and fiber densities. Preventive Medicine 1992; 21: 735-45.

27 Turrell G, Hewitt B, Patterson C, Oldenburg B, Gould T. Socioeconomic differences in food purchasing behaviour and suggested implications for diet-related health promotion. Journal of Human Nutrition and Dietetics 2002; 5 355-64.

28 Hodgson JM, Hsu-Hage B, Wahlqvist ML. Food variety as a quantitative descriptor of food intake. Ecology of Food and Nutrition 1994; 32: 137-48.

29 US Department of Agriculture. The Food Guide Pyramid. Home and Garden Bulletin No. 252. Washington, DC: Government Printing Office, 1992.

30 Dixon LB, Cronin FJ, Krebs-Smith SM. Let the pyramid guide your food choices: capturing the total diet concept. Journal of Nutrition 2001; 131: 461S-72S.

31 Australian Bureau of Statistics. National Health Survey: Users Guide. Catalogue No. 4363.0. Canberra: Australian Government Printing Service, 1995.

32 Australian Bureau of Statistics. 1995 National Nutrition Survey (NNS) Confidentialized Unit Record File (CURF). Canberra: Commonwealth of Australia, 1999.

33 Australian Bureau of Statistics. Australian National Nutrition Survey (1995). Catalogue No. 4801.0. Canberra: Australian Government Printing Service, 1997.

34 SPSS, Inc. SPSS for Windows, Version 11. Upper Saddle River, NJ: Prentice Hall, 2001.

35 Australian Bureau of Statistics (ABS). Census of Population and Housing. Canberra: ABS, 1996.

36 Australian Bureau of Statistics (ABS). Australian Social Trends 1997. Catalogue No. 4102.0. Canberra: ABS, 1997.

37 Australian Bureau of Statistics (ABS). Australian Social Trends 2002. Catalogue No. 4102.0. Canberra: ABS, 2002. 
38 Bourdieu P. Distinction: A Social Critique of the Judgement of Taste. London: Routledge \& Kegan Paul, 1984.

39 National Health and Medical Research Council (NHMRC). Dietary Guidelines for Australians - A Guide to Healthy Eating. Catalogue No. 0326099. Canberra: NHMRC, 2003.

40 Worsley A, Crawford D. Australian consumer acceptance of novel foods. Food Research Quarterly 1987; 47: 56-60.
41 Benton D. Carbohydrate ingestion, blood glucose and mood. Neuroscience and Biobehavioral Reviews 2002; 26: 293-308.

42 Thompson FE, Subar AF. Dietary assessment methodology. In: Coulston AM, Rock CL, Monsen ER, eds. Nutrition in the Prevention and Treatment of Disease. San Diego, CA: Academic Press, 2001. 